\title{
EDITORIAL \\ Outpatient surgery for brain tumours: A changing paradigm
}

The last couple of decades has witnessed an increasing trend in ambulatory surgery and day of admission surgery. Enhanced recovery after surgery (ERAS) is a multimodal perioperative pathway of care model designed to facilitate early discharge of patients from hospital and at the same time achieve improved functional capacity. ${ }^{[1]}$ The advantages to the patient are manifold and include less time spent in the hospital, less time lost at work preoperatively and the ability to remain in a familiar setting preoperatively, all of which lead to better patient satisfaction. Shortened hospital stays and earlier mobilisation also reduce the risk of hospital-acquired infections and venous thromboembolism and, interestingly, less risk for medical errors. ${ }^{[2-5]}$ Moreover, it is more economical to the hospital as there is more efficient and effective use of resources including decreased average length of stay and increased availability of inpatient surgical beds in the hospital. ${ }^{[6]}$ The latter leads to fewer case cancellations. ${ }^{[3]}$

Although it was routinely being done for spine surgery since the 1980s, ${ }^{[7]}$ there had been resistance amongst neurosurgeons in implementing ambulatory surgery for cranial procedures, primarily because these patients are at greater risk for adverse neurological outcomes and diminished long-term functional status as compared to the average surgical patient. Moreover, the occurrence of post-operative complications in patients following brain tumour surgery outside of a hospital setting could be devastating. With the advent of minimally invasive neurosurgery and image-guided surgery resulting in smaller openings, shorter recovery times and less post-operative pain, more and more centres in the West are now implementing ambulatory or same-day surgery admission for cranial neurosurgery, both awake and under general anaesthesia (GA). The Toronto group has been reporting the use of outpatient craniotomy since the last two decades, and the first pilot feasibility study was reported in 2001. ${ }^{[8]}$ Subsequently, they reported their 11-year experience with outpatient image-guided biopsies and outpatient craniotomy for intra-axial supratentorial tumours and concluded that it was safe and effective in selected patients. ${ }^{[5]}$ Initially, they were doing mainly awake craniotomies as outpatient surgery, but with more experience, they have expanded their protocol to include even craniotomies under GA. ${ }^{[3]}$ Subsequently, Grundy et al. reported the first UK series of day-case neurosurgery with similar results. ${ }^{[9]}$ Purzner et al. conducted a prospective analysis of 1003 patients over a 13-year period who underwent outpatient craniotomy, biopsy and spinal decompression. Out of the 249 patients who underwent craniotomy in this series, $92.8 \%$ were successfully discharged from the day surgery unit (DSU), 5.2\% were admitted from the DSU and 2.0\% were discharged and later readmitted. ${ }^{[4]}$

These authors did not report any adverse events as a result of outpatient surgery.

Patient selection is of utmost importance for successful implementation of outpatient surgery. In addition, patients and attendants need to be educated regarding the procedure, the expected complications and the necessity to readmit the patient in the event of complications. The post-operative complications that one is particularly concerned about in these patients include intracranial haematoma, post-operative brain swelling and seizures. The incidence of surgical haematoma following intracranial surgery requiring surgical evacuation is $2 \%{ }^{[10]}$ Taylor et al. found that intracranial haematoma usually occurs within $6 \mathrm{~h}$ of craniotomy or stereotactic biopsy. ${ }^{[11]}$ Keeping this in mind, the DSU protocol includes a post-operative computed tomography scan at $4 \mathrm{~h}$ and conversion to inpatient status in the event of significant haematoma or oedema, even if the patient is clinically asymptomatic. ${ }^{[1]}$ Usually, patients are discharged $6 \mathrm{~h}$ after surgery once the discharge criteria are met. Post-operative brain oedema, which usually peaks around the $2^{\text {nd }}$ or $3^{\text {rd }}$ post-operative day, has the incidence of $10 \% .^{[12]}$ Most neurosurgeons prefer to keep their patients under observation during this period although the value of this is not really known. ${ }^{[13]}$ In a recent 5-year retrospective observational study of same-day discharge for supratentorial tumours, Venkatraghavan et al. found that successful same-day discharge was possible in $88.4 \%$ of patient with a readmission rate of $2.9 \%{ }^{[1]}$ Same-day discharge has been found to be feasible after both GA and awake craniotomy. ${ }^{[1,3]}$ The key factors contributing to successful discharge on the day of surgery include careful patient selection on an individualised basis, small craniotomy flaps and an estimated surgical duration of $<4 \mathrm{~h}$. The latter would enable $6 \mathrm{~h}$ of post-operative monitoring before discharging the patient to home. Sughrue et al. demonstrated the feasibility of early discharge (day 1 or 2) following keyhole craniotomies under image guidance. ${ }^{[13]}$ In their series, $17 \%$ were infratentorial lesions. Their philosophy was to discharge 
patients the day after surgery once the discharge criteria were met. However, their readmission rate was higher $(4.8 \%)$, and $1.9 \%$ required readmission for repeat surgery to address complications. Their data suggested that the most serious post-operative complications following craniotomy usually occur within hours and not days and the complications requiring readmission would not have been avoided by longer inpatient stays. In fact, Venkatraghavan et al. ${ }^{[1]}$ found that the rate of readmission following one outpatient craniotomy $(2.9 \%)$ was similar to that following inpatient admission reported by Grundy et al ${ }^{[9]}$ The reasons for readmission cited by Au et al. include seizures, aphasia, wound haemostasis, cognitive impairment and new weakness. ${ }^{[3]}$ Of the $4.8 \%$ of readmissions in Sughrue's series, none were due to post-operative swelling. ${ }^{[13]}$ The incidence of post-operative seizures varies between $2 \%$ and $10 \%$ and usually occurs in the immediate post-operative period, ${ }^{[14]}$ and the necessity for observing patients for seizures beyond $6 \mathrm{~h}$ after surgery is doubtful. Most of the protagonists for outpatient surgery or early discharge for brain tumours are of the opinion that if the patient is stable the day after surgery, the risk of a catastrophic complication occurring thereafter was minimal. For an ERAS pathway to be successful, it should incorporate strict inclusion and exclusion criteria as well as discharge criteria. The latter should be a mutual decision amongst the patient, caregiver, neurosurgeon and neuroanaesthesiologist. Nurses also play a key role in facilitating outpatient surgery, especially with respect to providing information and education to patients and their caregivers, preparing them for surgery and helping them manage their post-operative care regarding medications, complications and precautions. ${ }^{[6]}$ Thus, in association with the physicians, they could take on the role of counsellor and address many concerns of the patient in the perioperative period. Khu assessed patients' perception of outpatient craniotomy for supratentorial tumours and they found that patient satisfaction was high and many patients were surprised that brain surgery can be done on an outpatient basis. ${ }^{[15]}$ These authors concluded that outpatient craniotomies made the disease less serious for the patient, contributed to their emotional well-being and could aid in the recovery process.

However, despite the medical, psychological and economic benefits, the concept of outpatient surgery for intracranial procedures has not gained momentum, particularly in India because of socioeconomic, medicolegal and ethical issues, and at present, there are no centres doing outpatient brain tumour surgery in India. ${ }^{[6]}$

Are we ready for implementing early discharge following craniotomies or stereotactic biopsies in our country? A recent article by Turel and Bernstein has addressed various issues and hurdles related to its adaptability in India. ${ }^{[6]}$ These authors have suggested that a good way to initiate it would be to start with early discharge on day 1 or 2 following surgery in selected patients and even selected hospitals and then determine whether this could be replicated in the outpatient setting.

\section{Mary Abraham}

Indian Spinal Injuries Centre, Vasant Kunj, New Delhi, India

Address for correspondence: Prof. Mary Abraham,

C-39D, Pocket C, Gangotri Enclave, Alaknanda, New Delhi, India. E-mail: doctormaryabraham@gmail.com

\section{REFERENCES}

1. Venkatraghavan L, Bharadwaj $S$, Au K, Bernstein $M$, Manninen P. Same-day discharge after craniotomy for supratentorial tumour surgery: A retrospective observational single-centre study. Can J Anaesth 2016;63:1245-57.

2. Pearse R, Dawson D, Fawcett J, Rhodes A, Grounds RM, Bennett ED. Early goal-directed therapy after major surgery reduces complications and duration of hospital stay. A randomised, controlled trial [ISRCTN38797445]. Crit Care 2005;9:R687-93.

3. Au K, Bharadwaj S, Venkatraghavan L, Bernstein $M$. Outpatient brain tumor craniotomy under general anesthesia. J Neurosurg 2016;125:1130-5.

4. Purzner T, Purzner J, Massicotte EM, Bernstein M. Outpatient brain tumor surgery and spinal decompression: A prospective study of 1003 patients. Neurosurgery 2011;69:119-26.

5. Boulton $\mathrm{M}$, Bernstein M. Outpatient brain tumor surgery: Innovation in surgical neurooncology. J Neurosurg 2008;108:649-54.

6. Turel MK, Bernstein M. Is outpatient brain tumor surgery feasible in India? Neurol India 2016;64:886-95.

7. Zahrawi F. Microlumbar discectomy. Is it safe as an outpatient procedure? Spine (Phila Pa 1976) 1994;19:1070-4.

8. Bernstein M. Outpatient craniotomy for brain tumor: A pilot feasibility study in 46 patients. Can J Neurol Sci 2001;28:120-4.

9. Grundy PL, Weidmann C, Bernstein M. Day-case neurosurgery for brain tumours: The early United Kingdom experience. $\mathrm{Br} \mathrm{J}$ Neurosurg 2008;22:360-7.

10. Khaldi A, Prabhu VC, Anderson DE, Origitano TC. The clinical significance and optimal timing of postoperative computed tomography following cranial surgery. J Neurosurg 2010;113:1021-5.

11. Taylor WA, Thomas NW, Wellings JA, Bell BA. Timing of postoperative intracranial hematoma development and implications for the best use of neurosurgical intensive care. J Neurosurg 1995;82:48-50.

12. Wong JM, Panchmatia JR, Ziewacz JE, Bader AM, Dunn IF, Laws ER, et al. Patterns in neurosurgical adverse events: Intracranial neoplasm surgery. Neurosurg Focus 2012;33:E16.

13. Sughrue ME, Bonney PA, Choi L, Teo C. Early discharge after surgery for intra-axial brain tumors. World Neurosurg 2015;84:505-10.

14. De Santis A, Villani R, Sinisi M, Stocchetti N, Perucca E. Add-on phenytoin fails to prevent early seizures after surgery for supratentorial brain tumors: A randomized controlled study. Epilepsia 2002;43:175-82. 
15. Khu KJ, Doglietto F, Radovanovic I, Taleb F, Mendelsohn D, Zadeh G, et al. Patients' perceptions of awake and outpatient craniotomy for brain tumor: A qualitative study. J Neurosurg 2010;112:1056-60.
This is an open access article distributed under the terms of the Creative Commons Attribution-NonCommercial-ShareAlike 3.0 License, which allows others to remix, tweak, and build upon the work non-commercially, as long as the author is credited and the new creations are licensed under the identical terms.

\begin{tabular}{|l|l|}
\hline \multicolumn{2}{|c|}{ Access this article online } \\
\hline Quick Response Code: & Website: \\
\hline & www.jnaccjournal.org \\
\cline { 2 - 2 } & \\
\hline
\end{tabular}

How to cite this article: Abraham M. Outpatient surgery for brain tumours: A changing paradigm. J Neuroanaesthesiol Crit Care 2017;4:1-3. 